\title{
CONTINUITY OF DERIVATIONS ON SOME SEMIPRIME BANACH ALGEBRA
}

\author{
RAMESH V. GARIMELLA
}

\begin{abstract}
If every prime ideal is closed in a commutative semiprime Banach algebra with unit, then every derivation on it is continuous. Also if derivations are continuous on integral domains, then they are continuous on semiprime Banach algebras.
\end{abstract}

1. Introduction. In [9] Singer and Wermer proved that the range of a continuous derivation on a commutative Banach algebra is contained in the Jacobson radical. They conjectured that the assumption of continuity is unnecessary. In [7] Johnson proved that if $A$ is a semisimple Banach algebra, then every derivation on $A$ is continuous and hence by the Singer-Wermer theorem it is zero.

In this note in $\S 3$, we prove that if $A$ is a semiprime Banach algebra in which every prime ideal is closed, then every derivation on $A$ is continuous. We also prove that if derivations are continuous on integral domains, then they are continuous on semiprime Banach algebras. In $\S 2$, we prove that if $\bigcap_{n \geq 1} R^{n}$ is contained in every closed prime ideal, then the separating ideal of every derivation is nilpotent, where $R$ is the Jacobson radical of $A$. This improves a result of [8]. We also note that if the Jacobson radical $R$ of $A$ is an integral domain and if there is a nonzero element $a \in R$ such that $\bigcap_{n \geq 1} a^{n} R=\{0\}$, then every derivation on $A$ is continuous, which generalizes a result of $[8]$.

Throughout the following we suppose $A$ is a commutative Banach algebra with unit. $R$ and $N$ will denote, respectively, the Jacobson and nil radicals of $A . N$ is also called the prime radical of $A$ and it consists of all the nilpotent elements of $A$. For any derivation $D$ on $A$, let

$$
S(D)=\left\{x \in A: \text { there are } x_{n} \rightarrow 0 \text { with } D x_{n} \rightarrow X\right\}
$$

be the separating ideal of $D$. Let

$$
A(S(D))=\{x \in A: x S(D)=0\}
$$

be the annihilator ideal of $S(D)$. By the closed graph theorem one can see that $D$ is continuous if and only if $S(D)=\{0\}$.

2. Even though the following is implicit in [8], we state and prove it as a separate lemma.

LEMMA 2.1. $S(D)$ is nilpotent if and only if $S(D) \cap R$ is nilpotent.

PROOF. One half of the proof is obvious. For the other half suppose that $S(D) \cap R$ is nilpotent. Then by Theorem (1) of [8], it follows that $D(A)$ is contained in $R$. Since $R$ is a closed ideal, it follows that $S(D) \subseteq \overline{D(A)} \subseteq R$. Q.E.D.

Received by the editors October 16, 1985.

1980 Mathematics Subject Classification (1985 Revision). Primary 46J05. 
REMARK 2.1. If $A$ has no nonmaximal closed prime ideals, then by Theorem 2.7 of [3], every separating ideal of a derivation is a nilpotent ideal. Thus if necessary we can always assume that $A$ has nonmaximal closed prime ideals.

THEOREM 2.1. If $\bigcap_{n \geq 1} R^{n}$ is contained in every closed prime ideal of $A$, then $S(D)$ is a nilpotent ideal.

ProOF. By Remark 2.1, we may assume that $A$ has nonmaximal closed prime ideals. By Lemma 2.1, it is enough to prove that $S(D) \cap R$ is nilpotent. Let $x \in S(D) \cap R$. Since $S(D)$ is a separating ideal, there is a positive integer $m$ such that $\overline{x^{m} S(D)}=\overline{x^{n} S(D)}$ for all $n \geq m$. It follows from the Mittag-Leffler Theorem $[1$, Theorem 3.3] and the hypothesis that

$$
\overline{x^{m} S(D)}=\bigcap_{n \geq 1} \overline{x^{n} S(D)}=\overline{\bigcap_{n \geq 1} x^{n} S(D)} \subseteq \overline{\bigcap_{n \geq 1} R^{n}} \subseteq P
$$

for every closed prime ideal $P$. Thus $x^{m+1}$ belongs to every closed prime ideal $P$ and hence so does $x$. Thus $S(D) \cap R$ is contained in $S(D) \cap P$ for every closed prime ideal $P$. But as noted in the proof of [3, Corollary 2.4], $S(D) \cap N=\bigcap(S(D) \cap P)$, where the intersection runs over all the minimal prime ideals $P$ (which are closed) such that $S(D) \nsubseteq P$. Hence by the above argument it follows that $S(D) \cap N=$ $S(D) \cap R$. Thus $S(D) \cap R$ is a closed nil ideal. Therefore $S(D) \cap R$ is a nilpotent ideal (cf. [5]). Q.E.D.

The following result is Lemma 3 of [8].

COROLlaRY 2.1. If $\bigcap_{n \geq 1} R^{n}=\{0\}$, then $S(D)$ is nilpotent.

REMARK 2.2. Let $D$ be a discontinuous derivation on $A$. Then by Theorem 2.7 of $[\mathbf{1}]$, it follows that there is an element $a \in A$ such that $D_{0}=a D$ is a discontinuous derivation on $A$ with the following property:

$$
\left\{\begin{array}{l}
\text { (i) } A\left(S\left(D_{0}\right)\right) \text { is a closed prime ideal of } A . \\
\text { (ii) For every } x \in A \text {, either } x \in A\left(S\left(D_{0}\right)\right) \text { or } \overline{x S\left(D_{0}\right)}=S\left(D_{0}\right) .
\end{array}\right.
$$

THEOREM 2.2. If the Jacobson radical $R$ is an integral domain and if there is an element $0 \neq a \in R$ such that $\bigcap_{n \geq 1} a^{n} R=\{0\}$, then every derivation on $A$ is continuous.

Proof. Suppose the theorem is false. Then by Remark 2.2 we may assume that there is a discontinuous derivation $D$ satisfying $(*)$, so that $A(S(D))$ is a prime ideal. First we prove that $S(D) \subseteq R$. Since $D$ is discontinuous by Lemma 2.1, $S(D) \cap R \neq\{0\}$. Let $0 \neq s \in S(D) \cap R$. Since $R$ is an integral domain, $s \notin A(S(D))$. Therefore by $(*), \overline{s(S(D))}=S(D)$. Since $R$ is a closed ideal, it follows that $S(D) \subseteq R$. Note that $S(D)$ is not contained in $A(S(D))$ and hence $R \nsubseteq A(S(D))$. Now consider $0 \neq a$ in $R$ such that $\bigcap_{n \geq 1} a^{n} R=\{0\}$. Note that $a \notin A(S(D)$ ). (For if $a \in A(S(D))$, then $a S(D)=\{0\}$. Since $R$ is an integral domain it follows that $a=0$.) Therefore by $(*), \overline{a S(D)}=S(D)$. Let $x \in S(D) \backslash A(S(D))$. Since $A(S(D))$ is a prime ideal, $a x \notin A(S(D))$. Therefore again by $(*)$ we get $x \in \overline{(a x S(D))}$. Hence $x \in \overline{a x R}$. Therefore by the equivalence conditions of class (iv) [4, p. 59], it follows that $\bigcap_{n \geq 1} a^{n} R \neq\{0\}$ which is a contradiction. Q.E.D.

The following result was proved in [8].

COROLlaRY 2.2. If $\bigcap_{n \geq 1} R^{n}=\{0\}$ and $R$ is an integral domain, then every derivation on $A$ is continuous. 
REMARK 2.3. Using the Mittag-Leffler Theorem [1, Theorem 3.3] and the same argument as that of Theorem 2.2, we can prove the following

THEOREM 2.3. Suppose $A$ is a commutative Banach algebra with unit which is also an integral domain. Further assume there is a nonzero closed ideal I such that $\bigcap_{n \geq 1} I^{n}=\{0\}$. Then every derivation on $A$ is continuous.

3. Following Khosravi [8], for any ideal $I$ of $A$, we define

$$
K(I)=\left\{x \in I: D^{n} x \in I \text { for all } n \geq 1\right\},
$$

where $D$ is any derivation on $A$.

The following lemma can be proved very easily and so we shall omit the proof.

LEMMA 3.1. For any ideal $I, K(I)$ is an ideal. Further $K(I)$ is a prime ideal if $I$ is a prime ideal.

REMARK 3.1. Let $A$ be a commutative semiprime Banach algebra with unit. Let $D: A \rightarrow A$ be a discontinuous derivation on $A$. Since $A$ is semiprime, as noted in the proof of Corollary 2.4 of [3], we get that $\bigcap(S(D) \cap P)=\{0\}$, where the instersection runs over all the minimal prime ideals $P$ such that $S(D) \nsubseteq P$. Now again by Theorem 2.7 of [1], there exists an element $a \in A$ such that $D_{0}=a D$ is a discontinuous derivation on $A$ satisfying (*) of Remark 2.2. Since $A$ is a semiprime Banach algebra, $S\left(D_{0}\right) \nsubseteq A\left(S\left(D_{0}\right)\right)$. Let $P$ be a minimal prime ideal such that $S(D) \nsubseteq P$. Since $S\left(D_{0}\right) \cdot A\left(S\left(D_{0}\right)\right)=\{0\}$, we get that either $S\left(D_{0}\right) \subseteq P$ or $A\left(S\left(D_{0}\right)\right) \subseteq P$. If $S\left(D_{0}\right)$ is contained in every such $P$, then since $S\left(D_{0}\right) \subseteq S(D)$, we get that $S\left(D_{0}\right)=\{0\}$, which is false. Therefore there exists a minimal prime ideal $P$ such that $S\left(D_{0}\right) \nsubseteq P$. Therefore $A\left(S\left(D_{0}\right) \subseteq P\right.$. Since $A\left(S\left(D_{0}\right)\right)$ is a prime ideal, we get $P=A\left(S\left(D_{0}\right)\right)$. Thus $A\left(S\left(D_{0}\right)\right)$ is a minimal prime ideal. Thus if necessary we can assume that there exists a discontinuous derivation $D$ such that $A(S(D))$ is a minimal prime ideal and for every $x \in A$ either $x \in A(S(D))$ or $\overline{x S(D)}=S(D)$.

THEOREM 3.1. The following conditions are equivalent.

(i) Every derivation on a commutative Banach algebra with unit which is an integral domain, is continuous.

(ii) Every derivation on a commutative semiprime Banach algebra with unit is continuous.

Proof. Obviously (ii) implies (i). So assume (i). Suppose (ii) is false. Then by Remark 3.1, there exists a semiprime Banach algebra $A$ with unit, and a discontinuus derivation $D$ satisfying (*) of Remark 2.2 and further $A(S(D))$ is a minimal prime ideal. Now by Lemma $3.1, K(A(S(D)))$ is a prime ideal of $A$ contained in $A(S(D))$. Since $A(S(D))$ is a minimal prime ideal we get that $A(S(D))=$ $K(A(S(D)))$. Therefore $A(S(D))$ is invariant under $D$. Hence $\tilde{D}: A / A(S(D)) \rightarrow$ $A / A(S(D))$ defined by $\tilde{D}(x+A(S(D)))=D x+A(S(D))$ is a derivation. Since $A / A(S(D))$ is an integral domain, by hypothesis, we get that $\tilde{D}$ is continuous. Hence $S(\tilde{D})=\{0\}$. This implies that $S(D) \subseteq A(S(D))$. Since $A$ is a semiprime Banach algebra we get that $S(D)=\{0\}$ which is a contradiction. This completes the proof of the theorem.

THEOREM 3.2. Let $A$ be a semiprime commutative Banach algebra with unit in which every prime ideal is closed. Then every derivation on $A$ is continuous. 
Proof. Suppose the theorem is false. We may assume that there is a discontinuous derivation $D$ satisfying the conditions mentioned in Remark 3.1.

Thus $A(S(D))$ is a minimal prime ideal and hence $K(A(S(D)))=A(S(D))$. In particular $A(S(D))$ is invariant under $D$ and we may lift $D$ to the integral domain $A / A(S(D))$. Note that the prime ideals of $A / A(S(D))$ are all closed. If the lifted derivation is discontinuous we may again assume that $(*)$ holds. Note that since we may now assume that $A$ is an integral domain, $A(S(D))=\{0\}$. Hence, if $I$ is a closed nonzero ideal and $0 \neq x \in I$, then $\overline{x S(D)}=S(D) \subseteq I$. Thus $S(D)$ is contained in every nonzero prime ideal. Also $S(D) \subseteq R$.

If $I$ is a nonzero ideal and if $S(x):=\left\{x, x^{2}, \ldots\right\}$ where $x \in S(D)$, then if $I \cap S(x)=\varnothing$, we can find a prime ideal $P \supseteq I$ with $S(x) \cap P=\varnothing$. But $P$ is a closed ideal and this contradicts the previous observation. Hence $S(x) \cap I \neq \varnothing$ for every nonzero ideal $I$ of $A$.

Now since $\overline{x S(D)}=S(D)$ for every $x \neq 0$, the argument of Theorem 8.1 of [4] applies and produces the existence of two elements $b, c \in S(D)$ such that $b^{n} \notin c A$ and $c^{n} \notin b A, n=1,2, \ldots$ This means $S(b) \cap c A=\varnothing$ and $S(c) \cap b A=\varnothing$. This contradicts the previous paragraph and proves that the lifted derivation must be continuous. Hence for the original derivation $D$, we obtain $S(D) \subseteq A(S(D))$, or $S(D)^{2}=\{0\}$. But $A$ is semiprime, so $S(D)=\{0\}$. This is a contradiction. Q.E.D.

FINAL REMARK. Banach algebras satisfying the hypothesis of Theorem 3.3 occur in the work of Sandy Grabiner [6] (in particular refer to Theorem 3.8, p. 176 and Theorem 3.15, p. 179). This was pointed out to me by the referee and also by Professor K. B. Laursen. I am very grateful to them. Finally, it is interesting to find some more examples of Banach algebras in which prime ideals are closed.

ACKNOWLEDGMENTS. I am very thankful to the referee for his valuable comments and suggestions in improving some proofs. Also I thank Dr. N. V. Rao for his encouragement.

\section{REFERENCES}

1. W. G. Bade and P. C. Curtis, Jr., Prime ideals and automatic continusity for Banach algebras, J. Funct. Anal. 29 (1978), 88-103.

2. Philip C. Curtis, Jr., Derivations on commutative Banach algebras (Proc., Long Beach, 1981), Lecture Notes in Math., vol. 975, Springer-Verlag, pp. 328-333.

3. J. Cusack, Automatic continuity and topologically simple radical Banach algebras, J. London Math. Soc. 21 (1977), 493-500.

4. J. Esterle, Elements for a classification of commutative radical Banach algebras (Proc., Long Beach, 1981), Lecture Notes in Math., vol. 975, Springer-Verlag, pp. 4-65.

5. S. Grabiner, The nilpotency of Banach nil algebras, Proc. Amer. Math. Soc. 21 (1969), 510.

6. __ A formal power series operational calculus for quasinilpotent operators. II, J. Math. Anal. Appl. 43 (1973), 170-192.

7. B. E. Johnson, Continuity of derivations on commutative algebras,, Amer. J. Math. 91 (1969), 1-10.

8. A. Khosravi, Derivations on commutative Banach algebras, Proc. Amer. Math. Soc. 84 (1982), 60-64.

9. M. Singer and J. Wermer, Derivatives on commutative normed algebras, Math. Ann. 129 (1955), 260-264.

Department of Mathematics, The University of Toledo, Toledo, Ohio 43606

Curnent address: Department of Mathematics and Statistics, Northwest Missouri State University, Maryville, Missouri 64468 